\title{
Genistein attenuates cancer stem cell characteristics in gastric cancer through the downregulation of Gli1
}

\author{
DAYEON YU ${ }^{1,2}$, HYUN-SOO SHIN ${ }^{3,4}$, YEO SONG LEE ${ }^{1,2}$, DOYEON LEE ${ }^{1}$, SHIN KIM $^{1,2}$ and YONG CHAN LEE ${ }^{1,2}$ \\ ${ }^{1}$ Department of Internal Medicine, Institute of Gastroenterology, ${ }^{2}$ Brain Korea 21 PLUS Project for Medical Science, \\ Yonsei University College of Medicine, Seoul; ${ }^{3}$ Division of Nephrology, Department of Internal Medicine, \\ ${ }^{4}$ Ewha Womans University School of Medicine, Ewha Medical Research Center, Seoul, Republic of Korea
}

Received August 26, 2013; Accepted October 9, 2013

DOI: $10.3892 /$ or.2013.2893

\begin{abstract}
Genistein is an isoflavone from soy with multiple action targets in cellular processes. Hedgehog signaling and its activator Gli1 are involved not only in oncogenesis, but also in cancer stemness and overexpression of CD44, a typical cancer stem cell surface marker. It has been shown that levels of Gli1 and CD44 expression are downregulated by genistein. Genistein may modulate distinctive cellular characteristics in cancer stem cells by inhibiting Gli1-related signaling pathways. In the present study, we sorted cells from MKN45, a human gastric cancer cell line, according to CD44 expression. CD44(+) cells showed properties of cancer stem-like cells and formed sphere colonies. In addition, sonic hedgehog (Shh) signaling genes were upregulated in CD44(+) cells when compared with these levels in CD44(-) cells. When CD44(+) cancer stem-like cells were treated with genistein, Gli1 and CD44 mRNA and protein expression was significantly reduced. Moreover, other stem cell markers were downregulated by genistein. Gli1 siRNA was used to confirm the action of genistein in inhibiting Gli1 expression. The high cell migration capacity of CD44(+) cells was blocked by genistein. In conclusion, genistein inhibits Gli1 gene expression, resulting in the attenuation of cancer stemlike properties in gastric cancer cells. In addition, genistein suppresses the cell invasive capacity that is required for tumor growth and metastasis. Our data showed that genistein can be an effective agent for gastric cancer therapy by targeting cancer stem-like characteristics.
\end{abstract}

\section{Introduction}

Genistein (4',5,7-trihydroxyisoflavone), one of the isoflavones derived from soybeans, exhibits many therapeutic effects,

Correspondence to: Professor Yong Chan Lee, Department of Internal Medicine, Yonsei University College of Medicine, 134 Shinchon-Dong, Seodaemun-Ku, Seoul 120-752, Republic of Korea E-mail: leeyc@yuhs.ac

Key words: genistein, gastric cancer, cancer stem cells, Gli1, CD44 particularly in anticancer treatment. Several epidemiological studies have reported that Asian women who consume more soy products when compared to Western women have a lower risk of breast cancer. This is based on the fact that breast cancer incidence in Asian women who have immigrated to Western countries is similar to that of Western women (1). It has also been shown that genistein inhibits cancer growth in many types of tumors in vitro and in vivo. Genistein pretreatment was found to lead to increased cancer cell death following cancer chemotherapy by inhibiting the activity of nuclear factor $\kappa \mathrm{B}$. Genistein was also found to regulate DNA methylation and histone modification $(2,3)$. The therapeutic action of genistein in gastric cancer is through suppression of the cancer cell growth via the apoptosis cascade (4). It was also found that genistein inhibits cell proliferation through cell cycle arrest in a dose-dependent manner (5).

Cancer stem cells are a subpopulation of cancer cells that have similar characteristics to normal stem cells. Cancer stem cells initiate new tumors, have self-renewing capacity and are pluripotent (6). The identification and management of cancer stem cells are important issues for targeted therapy in cancer treatment. In gastric cancer, cancer stem cells have not been well identified, but several studies have supported the existence of gastric cancer stem cells (7). CD44, a cell surface glycoprotein, is commonly used to isolate cancer stem cells in various types of tumors including gastric cancer (8-11).

Hedgehog $(\mathrm{Hh})$ signaling is essential for embryonic development and is responsible for maintaining many tissues and organs. Hh proteins [Sonic Hh (Shh), Indian $\mathrm{Hh}$ (Ihh) and Desert Hh (Dhh)] activate the transmembrane receptor Patched (Ptch). Ptch inhibits another transmembrane protein, Smoothened (Smo), and in the absence of Hh ligands, Hh signaling is blocked. Hh inhibits Ptch by binding to it, and as a result, the transcription factor Gli is activated $(12,13)$. There are three Gli proteins: Gli1, Gli2 and Gli3. Gli1 is a strong activator of targets, whereas Gli2 and Gli3 can be repressors or activators depending on the circumstance (14). Shh signaling is one of the important signaling pathways that have been implicated in human carcinogenesis. In addition, the Shh signaling pathway is reported to be crucial for maintaining the characteristics of cancer stem cells in gastric cancer (15).

In the present study, we investigated CD44(+) gastric cancer stem-like cells through the expression of stem cell- 
related genes and Shh signaling molecules. To determine the effect of genistein, we treated CD44(+) gastric cancer stemlike cells with genistein and analyzed gene expression changes and sphere-forming ability. Taken together, the present study evaluated the specific molecular targets by genistein in the Shh signaling pathway by attenuating gastric cancer stem cell characteristics.

\section{Materials and methods}

Cell culture. AGS (ATCC CRL 1739) and MKN45 (KCLB 80103) gastric cancer cells were maintained in RPMI-1640 medium (Thermo Fisher Scientific, Rockford, IL, USA) supplemented with $10 \%$ fetal bovine serum (FBS) (Thermo Fisher Scientific) and 1\% penicillin-streptomycin sulfate (Thermo Fisher Scientific). All cultures were maintained in a $37^{\circ} \mathrm{C}$ incubator supplemented with $5 \% \mathrm{CO}_{2}$.

Flow cytometric analysis and fluorescence-activated cell sorting $(F A C S)$. For cell surface marker analysis by flow cytometry, $\sim 80 \%$ confluent cells in a $100-\mathrm{mm}$ cell plate were washed with PBS, and then cells were detached from the plates using Trypsin-EDTA and centrifuged at $4^{\circ} \mathrm{C}$. Cell pellets were resuspended in HBSS (Gibco, Grand Island, NY, USA) supplemented with $1 \mathrm{mM}$ HEPES (Gibco) and 2\% FBS and filtered with a 40- $\mu$ m mesh filter (BD Biosciences, San Jose, CA, USA). The cells were stained with a 400-fold dilution of anti-CD44FITC (BD Biosciences) and incubated for $30 \mathrm{~min}$ in a $37^{\circ} \mathrm{C}$ incubator supplemented with $5 \% \mathrm{CO}_{2}$. The cells were then washed with HBSS and resuspended in HBSS supplemented with $1 \mathrm{mM}$ HEPES, 2\% FBS and 1\% penicillin-streptomycin sulfate. The cells were analyzed and sorted immediately with FACS Aria III (BD Biosciences).

Spheroid colony formation assay. FACS-sorted MKN45 cells were plated in each well of ultra-low-attachment 24-well plates (Corning Life Sciences, Corning, NY, USA) with DMEM/F12 medium (Thermo Fisher Scientific) supplemented with $20 \mathrm{ng} /$ ml EGF (R\&D Systems, Minneapolis, MN, USA), $10 \mathrm{ng} / \mathrm{ml}$ basic FGF (R\&D Systems), $1 \%$ insulin-transferrin-selenium (Gibco) and $1 \%$ penicillin-streptomycin sulfate. To investigate the effect of genistein, we added genistein to the assay media $(10 \mu \mathrm{g} / \mathrm{ml})$ and DMSO was used as the control. Every 3 days, each well was examined using a light microscope.

RT-PCR and real-time PCR. Total RNA was extracted from the cultured cells using TRIzol reagent (Invitrogen, Carlsbad, CA, USA) according to the manufacturer's instructions. First strand cDNA was synthesized using oligo(dT) primers and Superscript $^{\mathrm{TM}}$ II reverse transcriptase (Invitrogen). PCR was carried out with a PCR Maxi kit (Intron, Sungnam, Korea) according to the manufacturer's instructions. Amplification conditions included denaturation at $95^{\circ} \mathrm{C}$ for $15 \mathrm{~min}$, followed by 30 cycles of $30 \mathrm{sec}$ each at 95,55 and $72^{\circ} \mathrm{C}$. PCR products were separated on $2 \%$ agarose gels. Real-time PCR was carried out with a PCR mixture containing $1 \mu \mathrm{mol} / 1$ of each primer and SYBR-Green Master Mix (Applied Biosystems, Foster City, CA, USA). The amplifications were conducted at $95^{\circ} \mathrm{C}$ for $10 \mathrm{sec}$ and $60^{\circ} \mathrm{C}$ for $60 \mathrm{sec}$ using a StepOnePlus ${ }^{\mathrm{TM}}$ real-time PCR system (Applied Biosystems). Each sample was
Table I. Primer sequences

\begin{tabular}{lcl}
\hline Gene & Direction & \multicolumn{1}{c}{ Sequence (5'-3') } \\
\hline CD44 & F & GCTATTGAAAGCCTTGCAGAG \\
& R & CGCAGATCGATTTGAATATAACC \\
Oct4 & F & GACAACAATGAAAATCTTCAGGAGA \\
& R & TTCTGGCGCCGGTTACAGAACCA \\
Bmi & F & ATGTGTGTGCTTTGTGGAG \\
& R & AGTGGTCTGGTCTTGTGAAC \\
Nestin & F & AACAGCGACGGAGGTCTCTA \\
& R & TTCTCTTGTCCCGCAGACTT \\
ABCG2 & F & CTGAGATCCTGAGCCTTTGG \\
& R & TGCCCATCACAACATCATCT \\
Shh & F & GCAGAGTAGCCCTAACCGCT \\
& R & CAGGAGCCAGGTGCCTATTT \\
Ptch & F & CTCCCTACAGCAGCCACAGC \\
& R & AGGTCCCTTGTGGAGCRGGT \\
Gli1 & F & ACCCAGCCACCCCCTGATTA \\
& R & CCCCACCCTCCCACAGTAGC \\
B-actin & F & TTGCCGACAGGATGCAGAAG \\
& R & AGGTGGACAGCGAGGCCAGG \\
\hline
\end{tabular}

examined in triplicate, and the amount of PCR product was normalized with respect to $\beta$-actin as an internal control. PCR primers are shown in Table I.

Western blot analysis. Prepared cells were harvested after washing with PBS. Collected cells were lysed with buffer [50 mM Tris-Cl (pH 7.5), $150 \mathrm{mM} \mathrm{NaCl}, 1 \mathrm{mM}$ EDTA (pH 8.0), $1 \%$ Triton X-100, $1 \mathrm{mM}$ PMSF, $1 \mathrm{mM} \mathrm{Na}_{3} \mathrm{VO}_{4}$ and protease inhibitor cocktail (Roche Molecular Biochemicals, Indianapolis, IN, USA)]. The same amount of protein was boiled at $95^{\circ} \mathrm{C}$ after adding SDS sample buffer $[62.5 \mathrm{mM}$ Tris- $\mathrm{Cl}$ (pH 6.8), $2 \%$ SDS, $10 \%$ glycerol, $\beta$-mercaptoethanol, and $0.002 \%$ bromophenol blue]. Samples were loaded on $12 \%$ SDS-PAGE gels for Shh, Bmi and Oct4; 8\% SDS-PAGE gels for Ptch, Gli1, CD44, Nestin and ABCG2. Samples were transferred to PVDF membranes (Amersham Bioscience, Piscataway, NJ, USA).

Antibodies used included: Shh (1:1,000; Santa Cruz Biotechnology), Ptch1 (1:1,000; Abcam), Gli1 (1:1,000; Abcam), CD44 (1:1,000; Abcam), Oct4 (1:1,000; Santa Cruz Biotechnology), Bmi (1:1,000; Abcam), Nestin (1:1,000; Abcam), ABCG2 (1:1,000; Santa Cruz Biotechnology) and $\beta$-actin (1:2,000; Santa Cruz Biotechnology).

Immunofluorescence assay. Cells were grown on glass coverslips in a 6-well plate. After overnight incubation, the cells were washed with PBS three times, fixed in $10 \%$ formaldehyde for $10 \mathrm{~min}$ and permeabilized in $0.1 \%$ Triton X-100 in PBS for $3 \mathrm{~min}$. The cells were washed three times with PBS and blocked with $1 \%$ BSA in PBS buffer. The cells were incubated with the primary antibodies overnight at $4^{\circ} \mathrm{C}$. The slides were washed with PBS and incubated with FITC-conjugated 
Table II. Gli siRNA sequence.

\begin{tabular}{lc}
\hline Gene & Sequence \\
\hline Scramble siRNA & CGUACGCGGAAUACAACGA \\
Gli1siRNA & CUCCACAGGCAUACAGGAU
\end{tabular}

secondary antibody for $1 \mathrm{~h}$ at room temperature. The slides were mounted with DAPI. All samples were photographed using a Zeiss LSM 700 confocal microscope (Carl Zeiss, Oberkochen, Germany).

siRNA transfection. MKN45 cells were seeded onto 6-well plates and incubated overnight. The cells were transfected with siGli and scramble siRNA using Lipofectamine 2000 (Invitrogen) according to the manufacturer's instructions. After $48 \mathrm{~h}$, the cells were harvested and RNA and protein were isolated. siGlil and scramble siRNA sequences are shown in Table II.

Invasion assay. Transwells (24-well, $8.0 \mu \mathrm{m})$ (BD Biosciences) were coated with diluted Matrigel (BD Biosciences) in PBS and dried for $6 \mathrm{~h}$. The cells were resuspended in serum-free media, and then $4 \times 10^{5}$ cells were seeded in each top chamber. The lower chambers were filled with $10 \%$ FBS complete media for chemoattraction. To identify the effect of genistein, we added $10 \mu \mathrm{g} / \mathrm{ml}$ genistein and control DMSO to the top chambers. Cells were also transfected with siGlil and scramble siRNA for Glil knockdown. After $24 \mathrm{~h}$, the cells were seeded into the inserts. Cell invasion chambers were incubated for $48 \mathrm{~h}$ in a $37^{\circ} \mathrm{C}$ incubator supplemented with $5 \%$ $\mathrm{CO}_{2}$. Non-invaded cells on the upper surface were removed by a cotton swap, and the cells on the lower surface were fixed and stained using the Diff-Quik kit according to the manufacturer's instructions. The number of migrated cells on each insert was counted under a light microscope. Each sample was examined in triplicate.

\section{Results}

Genistein inhibits CD44 expression and other cancer stem cell markers. CD44 has been used as a cancer stem cell marker in many types of tumor cells including gastric cancer. To investigate the effect of genistein on the CD44(+) stem-like cell population, we incubated CD44(+) cells with $10 \mu \mathrm{g} / \mathrm{ml}$ genistein or DMSO as a control. After $72 \mathrm{~h}, \mathrm{CD} 44$ expression was analyzed by flow cytometry. CD44(+) cells decreased $\sim 40 \%$ in the genistein-treated CD44(+) cells when compared with the DMSO-treated CD44(+) control cells (Fig. 1A).

Formation of sphere colonies in serum-free media is one of the hallmarks of cancer stem cells. CD44(+) cells formed more spheres than CD44(-) cells in a time-dependent manner, and genistein significantly reduced the ability of sphere formation by CD44(+) gastric cancer cells (Fig. 1B).

Expression of cancer stem cell-related genes, Oct4, Bmi, Nestin and ABCG2, was determined at the mRNA level by RT-PCR and real-time PCR. We also examined the protein expression levels of several stemness-related genes, Oct4, Bmi,

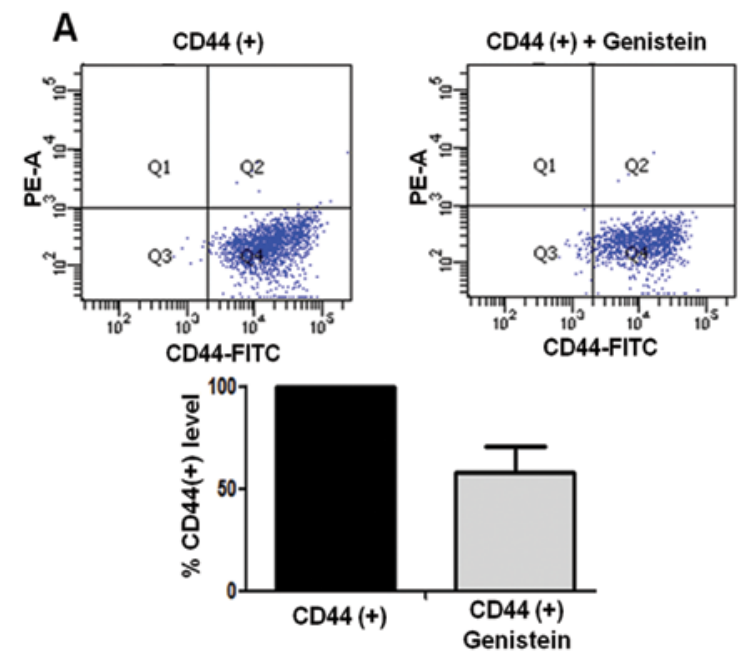

B
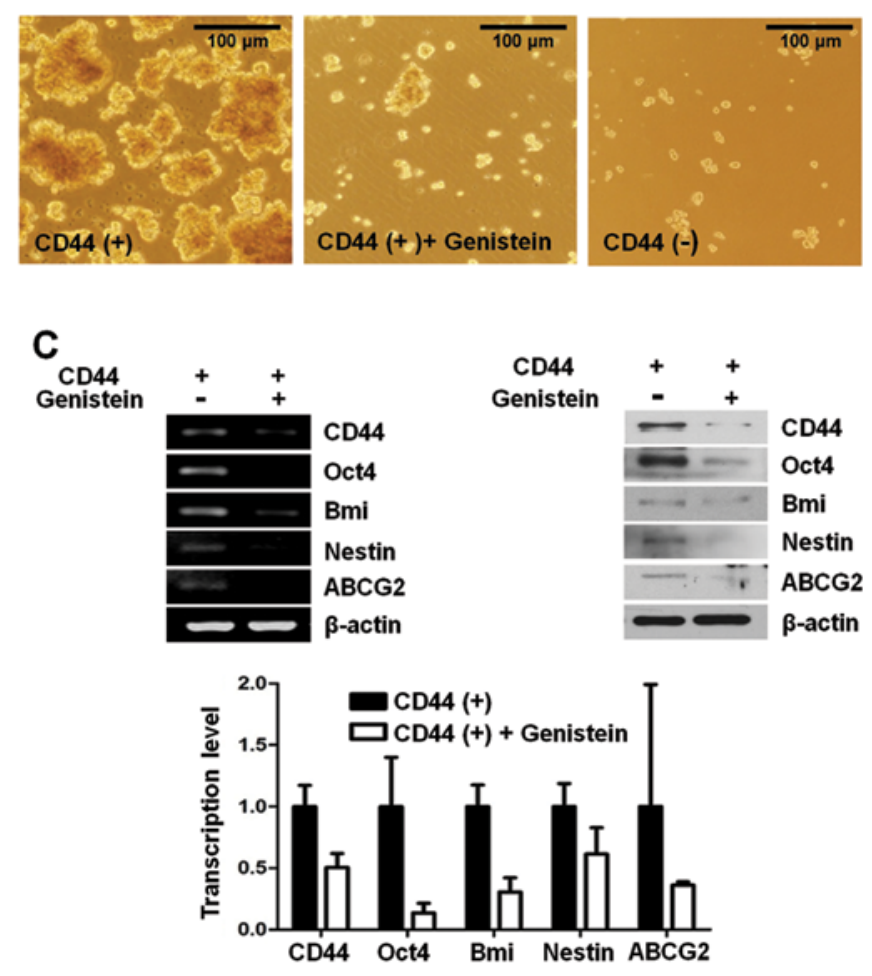

Figure 1. Genistein regulates expression of CD44 and stem cell-related genes. (A) Flow cytometric analysis of the expression of CD44 in FACS-sorted CD44(+) control and CD44(+) genistein-treated MKN45 groups. The cells were treated with $10 \mu \mathrm{g} / \mathrm{ml}$ genistein or control DMSO for $72 \mathrm{~h}$. The dot plots shown are from one representative experiment. The graph shows the CD44FITC mean value converted into a percentage. (B) Sphere formation assay in CD44(+), genistein-treated CD44(+) and CD44(-) MKN45 cells. The cells were treated with $10 \mu \mathrm{g} / \mathrm{ml}$ genistein or control DMSO, and the media were changed every 3 days. (C) Overexpression of cancer stem cell markers in CD44(+) gastric cancer stem-like MKN45 cells was suppressed by genistein. The cells were treated with $10 \mu \mathrm{g} / \mathrm{ml}$ genistein or control DMSO for $72 \mathrm{~h}$. RT-PCR, real-time PCR and western blot data revealed a decrease in the levels of these genes at the mRNA and protein levels.

Nestin and ABCG2, using western blot analysis in CD44(+) and CD44(-) cells. We found that the expression of these genes was significantly decreased in the genistein-treated CD44(+) cells when compared to these levels in the control CD44(+) cells at the mRNA and protein levels (Fig. 1C). 


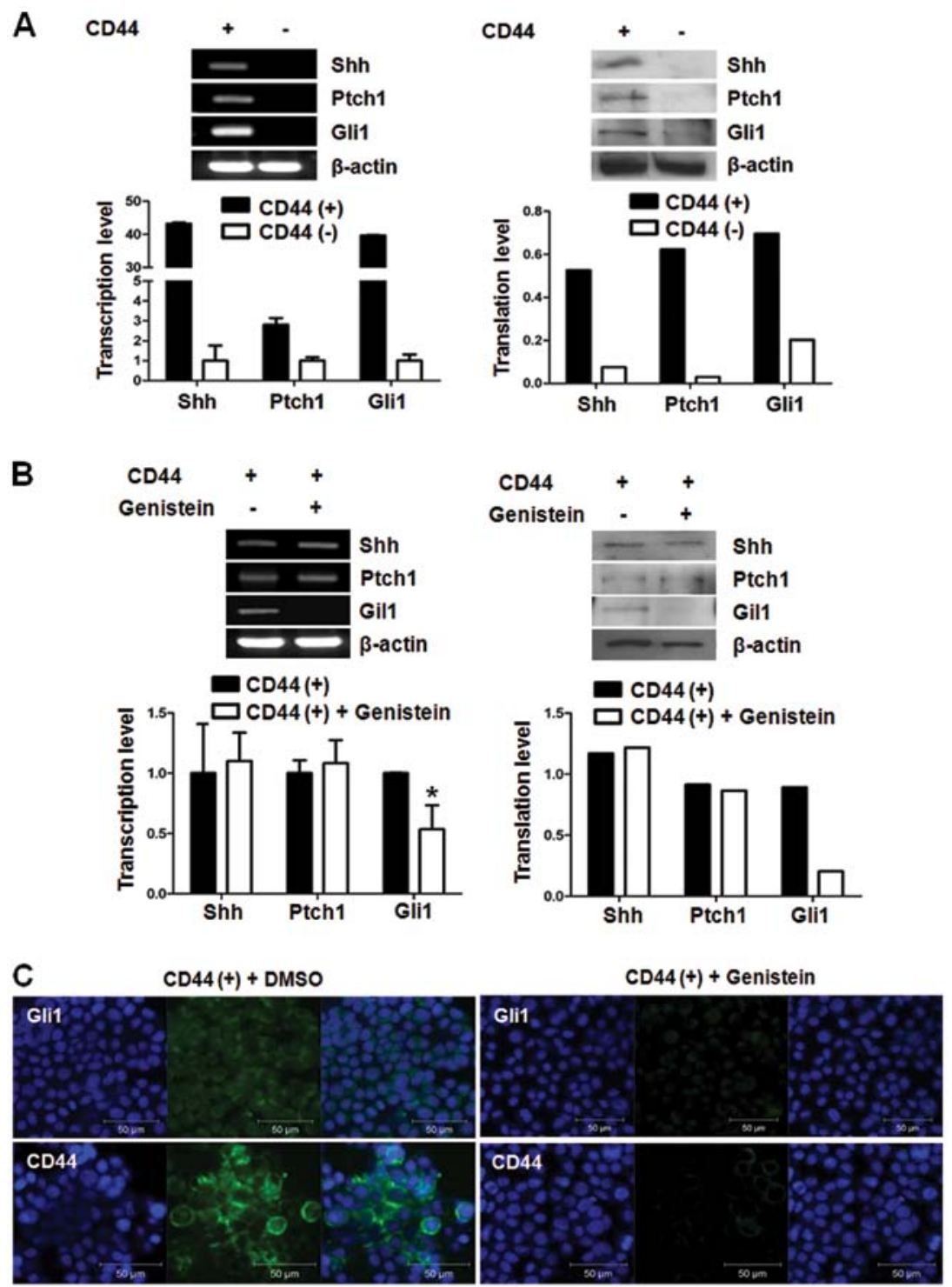

Figure 2. Inhibition of Shh signaling by genistein. (A) mRNA expression of Shh signaling genes: Shh, Ptch and Gli1 in CD44(+) and CD44(-) MKN45 cells was examined by RT-PCR and real-time PCR. Western blot data show their protein expression, and the histograms display the quantification of protein levels as compared to $\beta$-actin control. (B) Genistein inhibited Gli1 mRNA expression in CD44(+) MKN45 cells that had upregulated Shh signaling genes. Gli1 was decreased at the protein level while expression of other Shh signaling genes remained unchanged. The cells were treated with $10 \mu \mathrm{g} / \mathrm{ml} \mathrm{genistein} \mathrm{or} \mathrm{control}$ DMSO for $72 \mathrm{~h}$. (C) Immunofluorescence assay of CD44(+) control and genistein-treated MKN45 groups with anti-Gli1 (green, upper panel) and -CD44 (green, lower panel). Nuclei were stained with DAPI (blue).

Genistein suppresses the Shh signaling molecule, Glil. The Shh signaling pathway has been reported to be involved in cancer stem cell properties. Therefore, we performed RT-PCR and real-time PCR to identify the change in Shh signaling pathways and to validate the effect of Shh signaling on cancer stemness of CD44(+) cells at the mRNA level. CD44(+) cells showed upregulated expression of Shh, Ptch and Glil mRNA when compared to the expression in the CD44(-) cells. In addition, the protein levels of the Shh signaling genes were also overexpressed in the CD44(+) cells when compared to the protein levels in the CD44(-) cells (Fig. 2A).

We treated CD44(+) cells with genistein to confirm its inhibitory action on the Shh-Gli1 signaling pathway. We found that genistein downregulated only Gli1 at the mRNA level. Gli1 was also downregulated at the protein level (Fig. 2B). Based on these results, genistein affects the Shh signaling pathway through the inhibition of Gli1 expression in CD44(+) cells.

Gli1 and CD44 overexpression was confirmed by immunofluorescence assay. The results showed that the number of Gli1 and CD44 overexpressing cells in the CD44(+) cells were reduced in the genistein-treated CD44(+) cells (Fig. 2C).

Gli1-knockdown cells exhibit similar characteristics as genistein-treated cells and genistein inhibits the migration capacity of CD44(+) stem-like gastric cancer cells in vitro. We transfected Gli1 siRNA into CD44(+) cells to confirm the inhibitory effect of genistein on Glil expression in gastric cancer cells. CD44(+) cells were transfected with either scramble siRNA or Gli1 siRNA, and RNA and protein were isolated from cancer cells after $48 \mathrm{~h}$. The expression of cancer stem cell markers including CD44, Oct4, Bmi, Nestin and ABCG2 was 
A
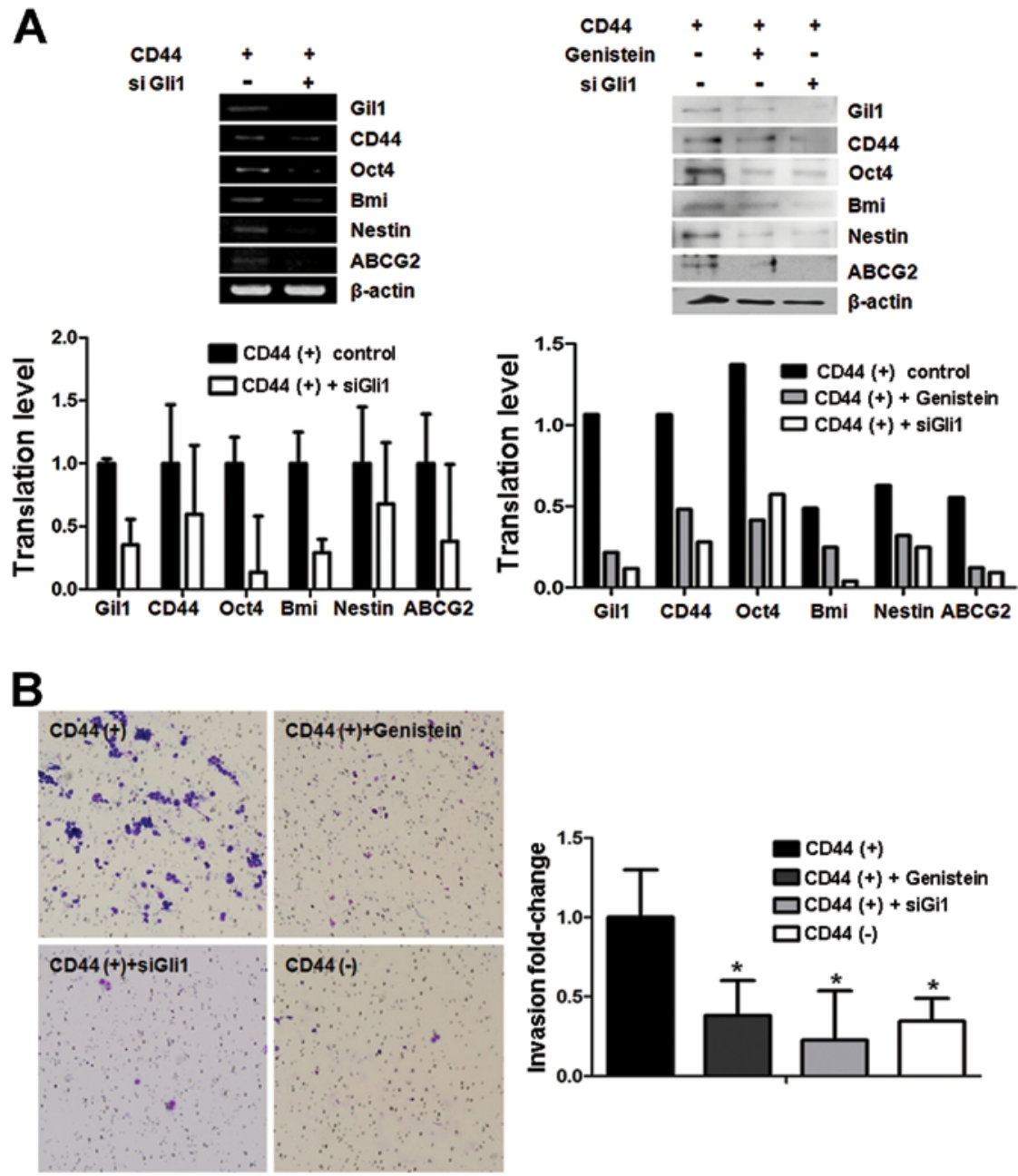

Figure 3. Gli1 knockdown affects expression of cancer stem-related genes, and genistein suppresses migratory capacity. (A) Gli1 knockdown in CD44(+) cells using siGlil caused a decrease in the expression of cancer stem-related genes at the mRNA and protein levels. The CD44(+) MKN45 cells were transfected with siGli1 and scramble siRNA for $72 \mathrm{~h}$. (B) Transwell invasion assays were conducted in two sets: CD44(+), CD44(-) control and genistein-treated CD44(+) cells; CD44(+), CD44(-) control and siGli1-transfected CD44(+) cells. Quantification of migrated cells indicated that CD44(+) cells were more invasive than CD44(-) cells $(\mathrm{P}<0.05)$. In addition, genistein and siGli1 significantly reduced cell migration $(\mathrm{P}<0.05)$.

assessed using real-time PCR and western blot analysis. The results showed that mRNA of cancer stem cell markers was decreased in the Gli1-downregulated CD44(+) cells by Gli1 siRNA pre-treatment. Following Glil knockdown, the changes in the protein expression levels of these markers in CD44(+) cells were validated by western blot analysis (Fig. 3A).

Migration and invasion properties are important characteristics of cancer stem cells responsible for tumor metastasis and growth. Cancer stem cells are assumed to have higher migration capacity than normal cancer cells. In addition, genistein at dietary concentrations can inhibit cancer invasion in vitro and in vivo (16). Genistein treatment reduced the invasive capacity of CD44(+) cells, and siGlil treatment showed a comparable effect in CD44(+) cells. The number of CD44(+) cells treated with siGli1 that had migrated was nearly $50 \%$ of the number of migratory $\mathrm{CD} 44(+)$ cells that were untreated, and the results were similar following genistein pre-treatment (Fig. 3B).

\section{Discussion}

In the present study, we investigated the feasibility of genistein as an anticancer molecule in gastric cancer, particularly for

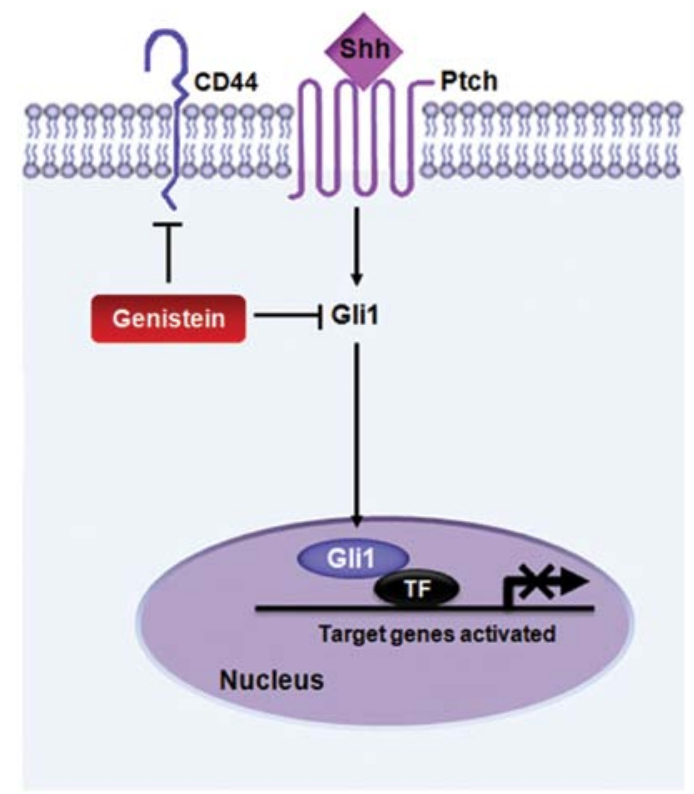

Figure 4. A schematic diagram of genistein action. Genistein inhibits the properties of gastric cancer stem cells though suppression of the Shh-Gli1 signaling pathway and CD44 expression. Shh, Sonic Hh; Ptch, Patched. 
targeting cancer stem cells. The present study was based on previous research that genistein induces suppression of the Hh signaling pathway $(17,18)$. We focused on the Shh-Gli1 pathway among many targets of genistein since Shh signaling is related to many of the characteristics noted in cancer stem cells. Cancer stem cells are considered to be a new target of cancer therapy as they have the potential to form new tumors and are difficult to kill due to their multidrug resistance potential (9). Therefore, inhibition of Gli1 to suppress cancer stemness would eliminate tumor cells completely. A previous report indicated that regulation of Hh-Glil signaling is a possible candidate for cancer therapy (19).

In the present study, cancer stem-like cells were identified in gastric cancer cell lines, AGS and MKN45, using CD44. CD44(+) cells exhibited upregulation of several cancer stem cell markers (data not shown) as well as the Shh-Glil signaling pathway and displayed high sphere colony forming ability. We found that cancer stem-like cells were enriched in the CD44(+) fraction when compared to the CD44(-) counterpart.

Next, we chose the appropriate concentration of genistein based on a cell proliferation assay and cell cytotoxicity assay to exclude effects through cell death and apoptotic damage (data not shown). After genistein treatment, Gli1 and CD44 expression was significantly decreased, and genistein affected the expression of cancer stem cell-related genes at both the transcriptional and translational levels. Moreover, our observations demonstrated that the Shh-Glil pathway is implicated in cancer stem cell properties. Glil knockdown in CD44(+) cells led to a reduction in the expression of stem cell-related genes, similar to that observed following genistein treatment. Cell invasion ability is highly maintained in cancer stem cells and this is strongly implicated in cancer growth and metastasis (20). Another important finding of our study was the effect of genistein on cell mobility. It has been demonstrated that genistein affects epithelial-mesenchymal transition (EMT) and cell invasion (21). We confirmed that genistein is an inhibitory agent for cancer invasion and found that Gli1 downregulation also affects the migratory capacity of cancer stem cells.

In summary, these data indicate that the soy isoflavone genistein controls Shh-Gli1 signaling and CD44 expression, and that it attenuates cancer stem cell properties (Fig. 4). We propose that genistein treatment may not only lead to cancer prevention, but that genistein itself could be used as an effective cancer therapy by modulating cancer stem cell characteristics.

\section{Acknowledgements}

The present study was supported by the Basic Science Research Program through the National Research Foundation of Korea (NRF) funded by the Ministry of Education, Science and Technology (grant no. NRF-2013R1A1A2009707 and NRF-2010-0024601).

\section{References}

1. Taylor CK, Levy RM, Elliott JC and Burnett BP: The effect of genistein aglycone on cancer and cancer risk: a review of in vitro, preclinical, and clinical studies. Nutr Rev 67: 398-415, 2009.

2. Zhang Y, Li Q and Chen H: DNA methylation and histone modifications of Wnt genes by genistein during colon cancer development. Carcinogenesis 34: 1756-1763, 2013.

3. Li Y, Ahmed F, Ali S, Philip PA, Kucuk O and Sarkar FH Inactivation of nuclear factor $\kappa \mathrm{B}$ by soy isoflavone genistein contributes to increased apoptosis induced by chemotherapeutic agents in human cancer cells. Cancer Res 65: 6934-6942, 2005.

4. Yanagihara K, Ito A, Toge T and Numoto M: Antiproliferative effects of isoflavones on human cancer cell lines established from the gastrointestinal tract. Cancer Res 53: 5815-5821, 1993.

5. Matsukawa Y, Marui N, Sakai T, et al: Genistein arrests cell cycle progression at G2-M. Cancer Res 53: 1328-1331, 1993.

6. Guo W, Lasky JL and Wu H: Cancer stem cells. Pediatr Res 59: 59R-64R, 2006.

7. Takaishi S, Okumura T and Wang TC: Gastric cancer stem cells. J Clin Oncol 26: 2876-2882, 2008.

8. Rocco A, Compare D and Nardone G: Cancer stem cell hypothesis and gastric carcinogenesis: experimental evidence and unsolved questions. World J Gastrointest Oncol 4: 54-59, 2012.

9. Du L, Wang H, He L, et al: CD44 is of functional importance for colorectal cancer stem cells. Clin Cancer Res 14: 6751-6760, 2008.

10. Zhang C, Li C, He F, Cai Y and Yang H: Identification of CD44+ CD2 $4^{+}$gastric cancer stem cells. J Cancer Res Clin Oncol 137: 1679-1686, 2011

11. Takaishi S, Okumura T, Tu S, et al: Identification of gastric cancer stem cells using the cell surface marker CD44. Stem Cells 27: 1006-1020, 2009.

12. Akiyoshi T, Nakamura M, Koga K, et al: Gli1, downregulated in colorectal cancers, inhibits proliferation of colon cancer cells involving Wnt signalling activation. Gut 55: 991-999, 2006.

13. Douard R, Moutereau S, Pernet P, et al: Sonic Hedgehogdependent proliferation in a series of patients with colorectal cancer. Surgery 139: 665-670, 2006.

14. Yanai K, Nagai S, Wada J, et al: Hedgehog signaling pathway is a possible therapeutic target for gastric cancer. J Surg Oncol 95: 55-62, 2007.

15. Song Z, Yue W, Wei B, et al: Sonic hedgehog pathway is essential for maintenance of cancer stem-like cells in human gastric cancer. PLoS One 6: e17687, 2011.

16. Pavese JM, Farmer RL and Bergan RC: Inhibition of cancer cell invasion and metastasis by genistein. Cancer Metastasis Rev 29: 465-482, 2010.

17. Ślusarz A, Shenouda NS, Sakla MS, et al: Common botanical compounds inhibit the hedgehog signaling pathway in prostate cancer. Cancer Res 70: 3382-3390, 2010.

18. Zhang L, Li L, Jiao M, et al: Genistein inhibits the stemness properties of prostate cancer cells through targeting Hedgehog-Gli1 pathway. Cancer Lett 23: 48-57, 2012.

19. Clement V, Sanchez P, de Tribolet N, Radovanovic I and Ruiz i Altaba A: HEDGEHOG-GLI1 signaling regulates human glioma growth, cancer stem cell self-renewal and tumorigenicity. Curr Biol 17: 165-172, 2007.

20. Singh A and Settleman J: EMT, cancer stem cells and drug resistance: an emerging axis of evil in the war on cancer. Oncogene 29: 4741-4751, 2010.

21. Zhang LL, Li L, Wu DP, et al: A novel anti-cancer effect of genistein: reversal of epithelial mesenchymal transition in prostate cancer cells. Acta Pharmacol Sin 29: 1060-1068, 2008. 